\title{
Microwave-Accelerated Click Chemistry: Expeditious Synthesis of Novel Triazole-linked Salicylic $\beta$-D-O-Glycosides with PTP1B Inhibitory Activity
}

\author{
Jin-Wei Yang, Cui Li, ${ }^{\ddagger}$ Xiao-Peng He, ${ }^{\ddagger}$, Hong Zhao, Li-Xin Gao,${ }^{\dagger}$ Wei Zhang, ${ }^{\dagger}$ Xiao-Xin Shi, ${ }^{\ddagger}$ Yun Tang,,${ }^{\ddagger}$ Jia Li, ${ }^{\dagger}, *$ \\ and Guo-Rong Chen*
}

\author{
Key Laboratory for Advanced Materials and Institute of Fine Chemicals, East China University of Science and Technology, \\ Shanghai 200237, P. R. China. ${ }^{*}$ E-mail: mrs guorongchen@ecust.edu.cn \\ ${ }^{\dagger}$ National Center for Drug Screening, Shanghai Institute of Materia Medica, Shanghai Institutes of Biological Sciences, \\ Chinese Academy of Sciences, Shanghai 201203, P. R. China. ${ }^{*}$ E-mail: jli@mail.shcnc.ac.cn \\ "School of Pharmacy, East China University of Science and Technology, Shanghai 200237, P. R. China \\ ${ }^{\S}$ PPSM, ENS Cachan, CNRS, 61 av President Wilson, F-94230 CACHAN, France \\ Received August 2, 2010, Accepted September 15, 2010
}

\begin{abstract}
The incorporation of microwave irradiation with the prevalent "click chemistry" is currently of considerable synthetic interest. We describe here the introduction of such laboratorial shortcut into carbohydrate-based drug discovery, resulting in the rapid formation of a series of triazole-linked salicylic $\beta$-D- $O$-glycosides with biological activities. All "clicked" products were achieved in excellent yields $(\approx 90 \%)$ within only a quarter. In addition, based on the structural characteristics of the afforded glycomimetics, their inhibitory activities were evaluated toward protein tyrosine phosphatases 1B (PTP1B) and a panel of homologous protein tyrosine phosphatases (PTPs). Docking simulation was also conducted to plausibly propose binding modes of this glycosyl salicylate series with the enzymatic target.
\end{abstract}

Key Words: Click chemistry, Microwave irradiation, Carbohydrate-based drug discovery, PTP1B inhibitor, Docking simulation

\section{Introduction}

Carbohydrates ubiquitously exist in nature and govern various biological and pathological events including cell-cell recognition, ${ }^{2}$ signal transduction ${ }^{3}$ and tumour metastasis. ${ }^{4}$ Considerable efforts have consequently been devoted by biologists toward the revelation of the substantial functions carbohydrates display as pivotal mediators in intricate cellular processes. ${ }^{5}$ On the other hand, numerous chemical approaches based on the synthesis of glycomimetics that possess great biological value have also been developed and proven indispensable as powerful allies for deciphering the genetic code that carbohydrates bear. ${ }^{1,6}$

Synthesized glycomimetics owning widely ranged biological applications could serve as, for example, biosensors, ${ }^{7}$ vaccines ${ }^{8}$ and glycan array substrates. ${ }^{9}$ Furthermore, in view of their intrinsic merits such as conformational flexibility, high biocompatibility and low toxicity, the abundantly affordable carbohydrates have been proposed as ideal scaffolds for sugar-based drug discovery. ${ }^{10,11}$ Such relatively untapped natural source do have subsequently provided exclusive opportunities for the advancement of small-molecule therapeutics. ${ }^{12}$ Hence, the development of potent and easily accessible synthetic methodologies for the efficient preparation of carbohydrate-based bioactive compounds leading to the facilitation of modern drug discovery has become quite intriguing.

The recently defined "click chemistry" of terminal alkynes with organic azides promoted by $\mathrm{Cu}(\mathrm{I})^{13}$ is a modular, selective and high yielding tool for organic synthesis. ${ }^{14}$ With these ideal features, it has been branded by Wong et al. as an excellent example for facilitating the fabrication of sugar-based bioactive compounds. ${ }^{15}$ Thus, the employment of click chemistry in such field has been continually delineated. For instance, triazolyl glycoconjugates were identified as human Fuc- $\mathrm{T},{ }^{15}$ carbonic anhydrase, ${ }^{16}$ glycosyltransferase,${ }^{17}$ and PTP1B inhibitors. $^{18 \mathrm{a}}$

Microwave irradiation was validated as a powerful assistant for enhancing the reactivity and tremendously economizing the reaction time in a broad area of organic synthesis. ${ }^{19}$ Its usage in click chemistry for combinatorial synthesis has also been successfully achieved ${ }^{20}$ However, though microwave-improved click chemistry leading to the accomplishment of functionalized glycomimetics are gradually being disclosed, ${ }^{21}$ reports on the rapid formation of sugar-based bioactive small molecules via such upgraded methodology remain scant. ${ }^{22}$

With a continuing interest on the construction of sugar-based bioactive compounds via click chemistry, ${ }^{18}$ we describe here the microwave-accelerated synthesis of a series of triazole-linked salicylic glycosides and their biological assessment. Our drug target is PTP1B (protein tyrosine phosphatase 1B), which represents a negative factor of type-2 diabetes, obesity and breast cancer. ${ }^{23}$ Because of the structural similarity between salicylate and pTyr (phosphotyrosine) substrate, salicylic derivatives have

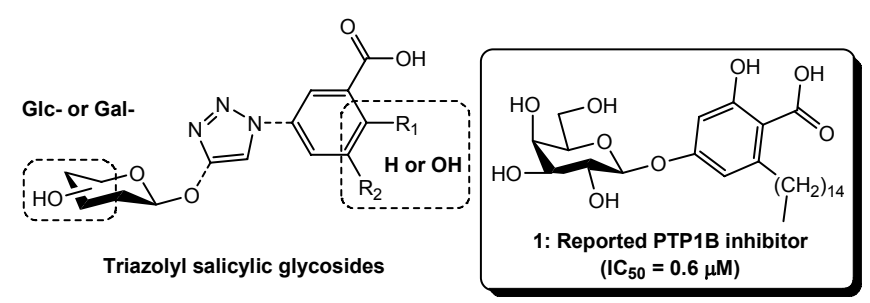

Figure 1. Salicylic $O$-glycoside derivatives as PTP1B inhibitors. 
been developed as potent competitive PTP1B inhibitors. ${ }^{24}$ Seo et al. also revealed in a recent study that a hydrolyzed natural $O$-galactoside (1, Fig. 1) displayed PTP1B inhibitory activity with the salicylic precursor being the critical pharmacophore. ${ }^{25}$

Prompted by such compelling evidence, we have designed and synthesized triazole-linked salicylic glycosides based on click chemistry (Fig. 1). Salicylic azides with position-varied or omitted hydroxyl group on benzoate ring were simultaneously prepared for SAR (structure-activity relationship) evaluation. Galactosyl and glucosyl alkynes were employed in this study for investigating the reactant tolerance and configuration impact of sugar moiety toward the bioactivity. The inhibitory activities of the afforded glycosyl compounds were subsequently assayed toward PTP1B and a panel of homologous PTPs. Docking simulation was also conducted to plausibly propose binding modes of this triazole-linked glycosyl salicylate series with the enzymatic target.

\section{Experimental Section}

General. All purchased chemicals and reagents were of high commercially available grade. Solvents were purified by standard procedures. ${ }^{1} \mathrm{H}$ and ${ }^{13} \mathrm{C}$ NMR spectrum were recorded on a Bruker AM-400 spectrometer in $\mathrm{CDCl}_{3}, \mathrm{D}_{2} \mathrm{O}$ or DMSO- $d_{6}$ solutions. Microwave-assisted reactions were performed in a Yalian (YL8023B1) system at $40{ }^{\circ} \mathrm{C}$ with a ramp time of $6 \mathrm{~min}$ and hold time of $8 \mathrm{~min}$. All reactions were monitored by TLC (thin-layer chromatography) with detection by UV or by spraying with $6 \mathrm{~N} \mathrm{H}_{2} \mathrm{SO}_{4}$ and charring at $300{ }^{\circ} \mathrm{C}$. Optical rotations were measured using a Perkin-Elmer 241 polarimeter at room temperature and a 10-cm 1-mL cell. High resolution mass spectra (HRMS) were recorded on a Waters LCT Premier XE spectrometer using standard conditions (ESI, $70 \mathrm{eV}$ ).

General procedure for the preparation of azides. To a soln. of salicylates (1 equiv.) in acetone $(10 \mathrm{~mL})$ and water $(10 \mathrm{~mL})$, were added $\mathrm{K}_{2} \mathrm{CO}_{3}$ (4 equiv.) and 1,2-dibromoethane (3 equiv.), stirring at $\mathrm{rt}$ for $6 \mathrm{~h}$. Upon completion, $\mathrm{NaN}_{3}$ (3 equiv.) was directly added and the mixture was stirred at $60^{\circ} \mathrm{C}$ for another $6 \mathrm{~h}$. The mixture was then washed with water, extracted with EtOAc and dried over $\mathrm{MgSO}_{4}$. After filtration and concentration, the crude residue was purified by column chromatography.

Methyl 3-(2-azidoethoxy)benzoate (5): From 2 (830 mg, 5.46 $\mathrm{mmol}$ ), column chromatography (EtOAc/petroleum ether, 1:1) afforded 5 as a colorless syrup (555.3 $\mathrm{mg}, 47.5 \%) . R_{f}=0.41$ (EtOAc/petroleum ether, 2:1); ${ }^{1} \mathrm{H}$ NMR $\left(400 \mathrm{MHz}, \mathrm{CDCl}_{3}\right) \delta$ $7.66(\mathrm{~d}, J=7.6 \mathrm{~Hz}, 1 \mathrm{H}), 7.55(\mathrm{~s}, 1 \mathrm{H}), 7.34(\mathrm{t}, J=7.8 \mathrm{~Hz}, 1 \mathrm{H})$, $7.12(\mathrm{dd}, J=2.4 \mathrm{~Hz}, 8.2 \mathrm{~Hz}, 1 \mathrm{H}), 4.16(\mathrm{t}, J=4.6 \mathrm{~Hz}, 2 \mathrm{H}), 3.89$ $(\mathrm{s}, 3 \mathrm{H}), 3.58(\mathrm{t}, J=4.6 \mathrm{~Hz}, 2 \mathrm{H}) ;{ }^{13} \mathrm{C} \mathrm{NMR}\left(100 \mathrm{MHz}, \mathrm{CDCl}_{3}\right) \delta$ 166.7, 158.2, 131.5, 129.5, 122.6, 120.0, 114.6, 67.1, 52.1, 50.1.

Methyl 3-(2-azidoethoxy)-5-hydroxybenzoate (6): From 3 (1600 mg, $9.51 \mathrm{mmol}$ ), column chromatography (EtOAc/petroleum ether, 1:1) afforded 6 as a yellow solid (973.9 mg, 50.1\%). $R_{f}=0.41$ (EtOAc/petroleum ether, 2:1); ${ }^{1} \mathrm{H} \mathrm{NMR}(400 \mathrm{MHz}$, $\left.\mathrm{CDCl}_{3}\right) \delta 7.18(\mathrm{~d}, J=7.2 \mathrm{~Hz}, 2 \mathrm{H}), 6.65(\mathrm{~s}, 1 \mathrm{H}), 5.63(\mathrm{~s}, 1 \mathrm{H})$, $4.16(\mathrm{t}, J=4.6 \mathrm{~Hz}, 2 \mathrm{H}), 3.90(\mathrm{~s}, 3 \mathrm{H}), 3.59(\mathrm{t}, J=3.6 \mathrm{~Hz}, 2 \mathrm{H}) ;{ }^{13} \mathrm{C}$ NMR $\left(100 \mathrm{MHz}, \mathrm{CDCl}_{3}\right) \delta 166.8,159.6,159.2,132.1,110.1$, 108.3, 107.2, 67.2, 52.4, 50.0.

Methyl 5-(2-azidoethoxy)-2-hydroxybenzoate (7): From 4
(1500 mg, $8.95 \mathrm{mmol}$ ), column chromatography (EtOAc/petroleum ether, $1: 1)$ afforded 7 as a yellow syrup (1002 mg, 52.3\%). $R_{f}=0.41$ (EtOAc/petroleum ether, 2:1); ${ }^{1} \mathrm{H} \mathrm{NMR}(400 \mathrm{MHz}$, $\left.\mathrm{CDCl}_{3}\right) \delta 7.31(\mathrm{~d}, J=3.1 \mathrm{~Hz}, 1 \mathrm{H}), 7.12(\mathrm{dd}, J=3.1 \mathrm{~Hz}, 9.0 \mathrm{~Hz}$, $1 \mathrm{H}), 6.93(\mathrm{~d}, J=9.0 \mathrm{~Hz}, 1 \mathrm{H}), 4.11(\mathrm{t}, J=5.0 \mathrm{~Hz}, 2 \mathrm{H}), 3.94(\mathrm{~s}$, $3 \mathrm{H}), 3.57(\mathrm{t}, J=5.0 \mathrm{~Hz}, 2 \mathrm{H}) ;{ }^{13} \mathrm{C}$ NMR $\left(100 \mathrm{MHz}, \mathrm{CDCl}_{3}\right) \delta$ $170.1,156.4,150.6,124.5,118.6,113.1,111.9,67.8,52.3,50.1$.

General procedure of microwave-assisted click reaction. To a soln. of sugar alkyne $(1.0 \mathrm{mmol})$ in $\mathrm{CH}_{2} \mathrm{Cl}_{2} / \mathrm{H}_{2} \mathrm{O}(10 \mathrm{~mL} /$ $10 \mathrm{~mL})$, were added azide $(1.2 \mathrm{mmol}), \mathrm{VcNa}(2.0 \mathrm{mmol})$ and $\mathrm{CuSO}_{4} \cdot 5 \mathrm{H}_{2} \mathrm{O}(1.0 \mathrm{mmol})$ which was then transferred to the microwave oven. After stirring for a ramp time of 6 min and heating time of $8 \mathrm{~min}$, the mixture was washed with sat $\mathrm{NaHCO}_{3}$, water and extracted with $\mathrm{CH}_{2} \mathrm{Cl}_{2}$ for three times. The combined organic layers were then dried over $\mathrm{MgSO}_{4}$, concentrated and purified by column chromatography.

Triazole tetra- $\boldsymbol{O}$-acetyl- $\boldsymbol{\beta}$-D- $\boldsymbol{O}$-glucosyl methyl benzoate (10): From 8 (50 mg, $0.13 \mathrm{mmol})$ and 5 (34.4 mg, $0.15 \mathrm{mmol})$, column chromatography (EtOAc/petroleum ether, 1:1) afforded 10 as a white powder $(69.4 \mathrm{mg}, 87.9 \%) . R_{f}=0.41$ (EtOAc/petroleum ether, $2: 1) ;[\alpha]_{\mathrm{D}}=-88.9\left(c 0.1, \mathrm{CH}_{2} \mathrm{Cl}_{2}\right) ;{ }^{1} \mathrm{HNMR}(400 \mathrm{MHz}$, $\left.\mathrm{CDCl}_{3}\right) \delta 7.72(\mathrm{~s}, 1 \mathrm{H}), 7.66(\mathrm{~d}, J=7.7 \mathrm{~Hz}, 1 \mathrm{H}), 7.51-7.50(\mathrm{~m}$, $1 \mathrm{H}), 7.36(\mathrm{t}, J=8.1 \mathrm{~Hz}, 1 \mathrm{H}), 7.07(\mathrm{dd}, J=2.6 \mathrm{~Hz}, 8.2 \mathrm{~Hz}, 1 \mathrm{H})$, $5.19(\mathrm{t}, J=9.4 \mathrm{~Hz}, 1 \mathrm{H}), 5.09(\mathrm{t}, J=9.7 \mathrm{~Hz}, 1 \mathrm{H}), 5.01(\mathrm{dd}, J=8.0$ $\mathrm{Hz}, 9.4 \mathrm{~Hz}, 1 \mathrm{H}), 4.94(\mathrm{~d}, J=12.6 \mathrm{~Hz}, 1 \mathrm{H}), 4.82(\mathrm{~d}, J=12.6 \mathrm{~Hz}$, $1 \mathrm{H}), 4.79(\mathrm{t}, J=4.9 \mathrm{~Hz}, 2 \mathrm{H}), 4.67(\mathrm{~d}, J=7.9 \mathrm{~Hz}, 1 \mathrm{H}), 4.41(\mathrm{t}$, $J=4.9 \mathrm{~Hz}, 2 \mathrm{H}), 4.26(\mathrm{dd}, J=4.7 \mathrm{~Hz}, 12.3 \mathrm{~Hz}, 1 \mathrm{H}), 4.14$ (dd, $J=2.2 \mathrm{~Hz}, 12.3 \mathrm{~Hz}, 1 \mathrm{H}), 3.89$ (s, 3H), 3.72-3.68 (m, 1H), 2.06 $(\mathrm{s}, 3 \mathrm{H}), 2.00(\mathrm{~s}, 3 \mathrm{H}), 1.97(\mathrm{~s}, 3 \mathrm{H}), 1.91(\mathrm{~s}, 3 \mathrm{H}) ;{ }^{13} \mathrm{C}$ NMR $(100$ $\left.\mathrm{MHz}, \mathrm{CDCl}_{3}\right) \delta 170.6,170.1,169.4,169.3,166.5,157.7,144.2$, 131.6, 129.7, 123.9, 123.0, 119.8, 114.7, 99.7, 72.7, 71.9, 71.2, 68.3, 66.4, 62.7, 61.8, 52.2, 49.7, 20.7, 20.5; HRMS: calcd. for $\mathrm{C}_{27} \mathrm{H}_{33} \mathrm{~N}_{3} \mathrm{O}_{13}+\mathrm{H}: 608.2092$, found: 608.2092 .

Triazole tetra- $O$-acetyl- $\beta$-D- $O$-glucosyl methyl 5-hydroxybenzoate (11): From 8 (50 mg, $0.13 \mathrm{mmol}$ ) and 6 (35.6 mg, 0.15 mmol), column chromatography (EtOAc/petroleum ether, 1:1) afforded 11 as a white powder $(75.8 \mathrm{mg}, 93.6 \%) . R_{f}=0.26$ (EtOAc/petroleum ether, 3:1); $[\alpha]_{\mathrm{D}}=-61.0\left(c 0.1, \mathrm{CH}_{2} \mathrm{Cl}_{2}\right)$; ${ }^{1} \mathrm{H}$ NMR $\left(400 \mathrm{MHz}, \mathrm{CDCl}_{3}\right) \delta 7.73(\mathrm{~s}, 1 \mathrm{H}), 7.19$ (brs, $\left.1 \mathrm{H}\right), 7.06$ (brs, $1 \mathrm{H}), 6.56(\mathrm{t}, J=1.9 \mathrm{~Hz}, 1 \mathrm{H}), 5.20(\mathrm{t}, J=9.4 \mathrm{~Hz}, 1 \mathrm{H}), 5.12$ $(\mathrm{t}, J=9.4 \mathrm{~Hz}, 1 \mathrm{H}), 5.06-5.02(\mathrm{~m}, 1 \mathrm{H}), 4.98(\mathrm{~d}, J=12.6 \mathrm{~Hz}, 1 \mathrm{H})$, $4.84(\mathrm{~d}, J=12.7 \mathrm{~Hz}, 1 \mathrm{H}), 4.78-4.68(\mathrm{~m}, 2 \mathrm{H}), 4.64(\mathrm{~d}, J=7.9 \mathrm{~Hz}$, 1H), 4.41-4.32 (m, 2H), 4.27 (dd, $J=4.6 \mathrm{~Hz}, 12.3 \mathrm{~Hz}, 1 \mathrm{H}), 4.15$ (dd, $J=1.6 \mathrm{~Hz}, 12.3 \mathrm{~Hz}, 1 \mathrm{H}), 3.88(\mathrm{~s}, 3 \mathrm{H}), 3.73-3.70(\mathrm{~m}, 1 \mathrm{H})$, $2.07(\mathrm{~s}, 3 \mathrm{H}), 2.01(\mathrm{~s}, 3 \mathrm{H}), 2.00(\mathrm{~s}, 3 \mathrm{H}), 1.96(\mathrm{~s}, 3 \mathrm{H}) ;{ }^{13} \mathrm{C} \mathrm{NMR}$ $\left(100 \mathrm{MHz} \mathrm{CDCl}_{3}\right) \delta 170.8,170.3,169.7,169.5,166.6,158.7$, $158.1,143.9,132.1,124.4,110.5,107.1,106.5,99.5,72.7,71.7$, 71.2, 68.2, 66.3, 62.3, 61.7, 52.2, 49.7, 20.6, 20.4; HRMS: calcd. For $\mathrm{C}_{27} \mathrm{H}_{33} \mathrm{~N}_{3} \mathrm{O}_{14}+\mathrm{H}: 624.2041$, found: 624.2040.

Triazole tetra- $O$-acetyl- $\beta$-D- $O$-glucosyl methyl 2-hydroxybenzoate (12): From 8 (50 mg, $0.13 \mathrm{mmol}$ ) and 6 (35.6 mg, 0.15 $\mathrm{mmol}$ ), column chromatography (EtOAc/petroleum ether, $1: 1$ ) afforded 12 as a white powder $(71.3 \mathrm{mg}, 88.0 \%) . R_{f}=0.53$ (EtOAc/petroleum ether, 3:1); $[\alpha]_{\mathrm{D}}=-33.6\left(c 0.1, \mathrm{CH}_{2} \mathrm{Cl}_{2}\right)$; ${ }^{1} \mathrm{H} \mathrm{NMR}\left(400 \mathrm{MHz}, \mathrm{CDCl}_{3}\right) \delta 10.39(\mathrm{~s}, 1 \mathrm{H}), 7.71(\mathrm{~s}, 1 \mathrm{H}), 7.25$ $(\mathrm{d}, J=3.1 \mathrm{~Hz}, 1 \mathrm{H}), 7.05(\mathrm{dd}, J=3.1 \mathrm{~Hz}, 9.0 \mathrm{~Hz}, 1 \mathrm{H}), 6.92(\mathrm{~d}$, $J=9.0 \mathrm{~Hz}, 1 \mathrm{H}), 5.19$ (t, $J=9.4 \mathrm{~Hz}, 1 \mathrm{H}), 5.10(\mathrm{t}, J=9.7 \mathrm{~Hz}, 1 \mathrm{H})$, $5.02(\mathrm{dd}, J=8.0 \mathrm{~Hz}, 9.4 \mathrm{~Hz}, 1 \mathrm{H}), 4.95(\mathrm{~d}, J=12.6 \mathrm{~Hz}, 1 \mathrm{H}), 4.83$ 
$(\mathrm{d}, J=12.6 \mathrm{~Hz}, 1 \mathrm{H}), 4.74(\mathrm{t}, J=4.9 \mathrm{~Hz}, 2 \mathrm{H}), 4.67(\mathrm{~d}, J=8.0$ $\mathrm{Hz}, 1 \mathrm{H}), 4.32(\mathrm{t}, J=4.9 \mathrm{~Hz}, 2 \mathrm{H}), 4.27(\mathrm{dd}, J=4.7 \mathrm{~Hz}, 12.3 \mathrm{~Hz}$, $1 \mathrm{H}), 4.14$ (dd, $J=2.2 \mathrm{~Hz}, 12.3 \mathrm{~Hz}, 1 \mathrm{H}), 3.93(\mathrm{~s}, 3 \mathrm{H}), 3.72-3.68$ (m, 1H), $2.07(\mathrm{~s}, 3 \mathrm{H}), 2.00(\mathrm{~s}, 3 \mathrm{H}), 1.97(\mathrm{~s}, 3 \mathrm{H}), 1.92(\mathrm{~s}, 3 \mathrm{H}) ;{ }^{13} \mathrm{C}$ NMR $\left(100 \mathrm{MHz} \mathrm{CDCl}_{3}\right) \delta 170.5,170.0,169.9,169.3,169.2$, $156.5,150.0,144.1,124.3,123.9,118.7,113.1,111.9,99.6$, 72.6, 71.7, 71.1, 68.2, 67.0, 62.7, 61.7, 52.3, 49.7, 20.6, 20.5; HRMS: calcd. For $\mathrm{C}_{27} \mathrm{H}_{33} \mathrm{~N}_{3} \mathrm{O}_{14}+\mathrm{H}: 624.2041$, found: 624.2039.

Triazole tetra- $\boldsymbol{O}$-acetyl- $\beta$-D- $\boldsymbol{O}$-galactosyl methyl benzoate (13): From compound 9 (50 mg, $0.13 \mathrm{mmol})$ and 5 (34.4 mg, $0.15 \mathrm{mmol}$ ), column chromatography (EtOAc/petroleum ether, $1: 1)$ afforded 13 as a white powder $(68.4 \mathrm{mg}, 86.7 \%) . R_{f}=0.32$ (EtOAc/petroleum ether, 2:1); $[\alpha]_{\mathrm{D}}=-44.2\left(c 0.1, \mathrm{CH}_{2} \mathrm{Cl}_{2}\right)$; ${ }^{1} \mathrm{H}$ NMR $\left(400 \mathrm{MHz}, \mathrm{CDCl}_{3}\right) \delta 7.72(\mathrm{~s}, 1 \mathrm{H}), 7.66(\mathrm{~d}, J=7.7$ $\mathrm{Hz}, 1 \mathrm{H}), 7.51$ (brs, $1 \mathrm{H}), 7.36(\mathrm{t}, J=8.0 \mathrm{~Hz}, 1 \mathrm{H}), 7.08$ (dd, $J=$ $2.1 \mathrm{~Hz}, 8.0 \mathrm{~Hz}, 1 \mathrm{H}), 5.38(\mathrm{~d}, J=3.2 \mathrm{~Hz}, 1 \mathrm{H}), 5.23(\mathrm{dd}, J=8.0$ $\mathrm{Hz}, 10.4 \mathrm{~Hz}, 1 \mathrm{H}), 5.00$ (dd, $J=3.4 \mathrm{~Hz}, 10.5 \mathrm{~Hz}, 1 \mathrm{H}), 4.98$ (d, $J=12.6 \mathrm{~Hz}, 1 \mathrm{H}), 4.82(\mathrm{~d}, J=13.4 \mathrm{~Hz}, 1 \mathrm{H}), 4.79(\mathrm{t}, J=5.2 \mathrm{~Hz}$, $2 \mathrm{H}), 4.64(\mathrm{~d}, J=7.9 \mathrm{~Hz}, 1 \mathrm{H}), 4.41(\mathrm{t}, J=4.8 \mathrm{~Hz}, 2 \mathrm{H}), 4.18-4.10$ $(\mathrm{m}, 2 \mathrm{H}), 3.94(\mathrm{t}, J=6.7 \mathrm{~Hz}, 1 \mathrm{H}), 3.89(\mathrm{~s}, 3 \mathrm{H}), 2.13(\mathrm{~s}, 3 \mathrm{H}), 2.04$ $(\mathrm{s}, 3 \mathrm{H}), 1.95(\mathrm{~s}, 3 \mathrm{H}), 1.92(\mathrm{~s}, 3 \mathrm{H}) ;{ }^{13} \mathrm{C} \mathrm{NMR}\left(100 \mathrm{MHz}, \mathrm{CDCl}_{3}\right)$ $\delta 170.3,170.1,169.9,169.4,166.4,157.7,144.1,131.5,129.6$, $123.9,122.8,119.6,114.6,100.1,70.7,68.6,67.0,66.4,62.6$, 61.2, 52.2, 49.6, 20.6, 20.5; HRMS: calcd. for $\mathrm{C}_{27} \mathrm{H}_{33} \mathrm{~N}_{3} \mathrm{O}_{13}+\mathrm{H}$ : 608.2092, found: 608.2090 .

Triazole tetra- $O$-acetyl- $\beta$-D- $O$-galactosyl methyl 5-hydroxybenzoate (14): From compound $9(50 \mathrm{mg}, 0.13 \mathrm{mmol})$ and 6 (35.6 mg, $0.15 \mathrm{mmol}$ ), column chromatography (EtOAc/petroleum ether, 1:1) afforded 14 as a white powder $(76.6 \mathrm{mg}, 94.5 \%)$. $R_{f}=0.19($ EtOAc/petroleum ether, $1: 1) ;[\alpha]_{\mathrm{D}}=-14.0(c 0.1$, $\left.\mathrm{CH}_{2} \mathrm{Cl}_{2}\right) ;{ }^{1} \mathrm{H} \mathrm{NMR}\left(400 \mathrm{MHz}, \mathrm{CDCl}_{3}\right) \delta 7.77$ (brs, $\left.1 \mathrm{H}\right), 7.19(\mathrm{~s}$, $1 \mathrm{H}), 7.05(\mathrm{~s}, 1 \mathrm{H}), 6.58(\mathrm{~s}, 1 \mathrm{H}), 5.39(\mathrm{~d}, J=3.3 \mathrm{~Hz}, 1 \mathrm{H}), 5.25$ $(\mathrm{dd}, J=7.9 \mathrm{~Hz}, 10.4 \mathrm{~Hz}, 1 \mathrm{H}), 5.03(\mathrm{dd}, J=3.4 \mathrm{~Hz}, 10.4 \mathrm{~Hz}, 1 \mathrm{H})$, $5.00(\mathrm{~d}, J=12.7 \mathrm{~Hz}, 1 \mathrm{H}), 4.83-4.70(\mathrm{~m}, 3 \mathrm{H}), 4.63(\mathrm{~d}, J=7.9 \mathrm{~Hz}$, $1 \mathrm{H}), 4.40-4.32(\mathrm{~m}, 2 \mathrm{H}), 4.19-4.09(\mathrm{~m}, 2 \mathrm{H}), 3.95(\mathrm{t}, J=6.5 \mathrm{~Hz}$, $1 \mathrm{H}), 3.87(\mathrm{~s}, 3 \mathrm{H}), 2.14(\mathrm{~s}, 3 \mathrm{H}), 2.04(\mathrm{~s}, 3 \mathrm{H}), 1.97(\mathrm{ds}, 6 \mathrm{H}) ;{ }^{13} \mathrm{C}$ NMR $\left(100 \mathrm{MHz} \mathrm{CDCl}_{3}\right) \delta 170.6,170.3,170.2,169.9,166.6$, 158.7, 158.1, 132.1, 110.4, 107.1, 106.6, 100.0, 70.8, 70.7, 68.8, 67.0, 66.4, 62.4, 61.2, 52.2, 49.8, 20.6, 20.5; HRMS: calcd. For $\mathrm{C}_{27} \mathrm{H}_{33} \mathrm{~N}_{3} \mathrm{O}_{14}+\mathrm{H}: 624.2041$, found: 624.2042 .

Triazole tetra- $\boldsymbol{O}$-acetyl- $\beta$-D- $\boldsymbol{O}$-galactosyl methyl 2-hydroxybenzoate (15): From compound 9 (50 $\mathrm{mg}, 0.13 \mathrm{mmol})$ and 7 (35.6 mg, $0.15 \mathrm{mmol}$ ), column chromatography (EtOAc/petroleum ether, 1:1) afforded 15 as a white powder (74.6 mg, 92.1\%). $R_{f}=0.28($ EtOAc/Dichloromethane, $1: 2) ;[\alpha]_{\mathrm{D}}=-79.2(c 0.1$, $\left.\mathrm{CH}_{2} \mathrm{Cl}_{2}\right) ;{ }^{1} \mathrm{H} \mathrm{NMR}\left(400 \mathrm{MHz}, \mathrm{CDCl}_{3}\right) \delta 10.40(\mathrm{~s}, 1 \mathrm{H}), 7.76$ (brs, $1 \mathrm{H}), 7.06(\mathrm{dd}, J=3.0 \mathrm{~Hz}, 9.0 \mathrm{~Hz}, 1 \mathrm{H}), 6.92(\mathrm{~d}, J=9.0 \mathrm{~Hz}, 1 \mathrm{H})$, $5.39(\mathrm{~d}, J=3.2 \mathrm{~Hz}, 1 \mathrm{H}), 5.24(\mathrm{dd}, J=7.9 \mathrm{~Hz}, 10.3 \mathrm{~Hz}, 1 \mathrm{H}), 5.01$ (dd, $J=3.4 \mathrm{~Hz}, 10.4 \mathrm{~Hz}, 1 \mathrm{H}), 4.95-4.80(\mathrm{~m}, 2 \mathrm{H}), 4.74$ (t, $J=$ $4.5 \mathrm{~Hz}, 2 \mathrm{H}), 4.65(\mathrm{~d}, J=7.9 \mathrm{~Hz}, 1 \mathrm{H}), 4.32(\mathrm{t}, J=4.7 \mathrm{~Hz}, 2 \mathrm{H})$, 4.19-4.08 (m, 3H), 3.94 (s, 3H), $2.13(\mathrm{~s}, 3 \mathrm{H}), 2.04(\mathrm{~s}, 3 \mathrm{H}), 1.96$ $(\mathrm{s}, 3 \mathrm{H}), 1.93(\mathrm{~s}, 3 \mathrm{H}) ;{ }^{13} \mathrm{C}$ NMR $\left(100 \mathrm{MHz}, \mathrm{CDCl}_{3}\right) \delta 170.3$, 170.2, 170.0, 169.9, 169.4, 156.6, 150.1, 124.4, 118.8, 113.1, 111.9, 100.3, 70.8, 68.7, 67.1, 67.0, 62.7, 61.2, 52.4, 49.9, 20.6, 20.5; HRMS: calcd. For $\mathrm{C}_{27} \mathrm{H}_{33} \mathrm{~N}_{3} \mathrm{O}_{14}+\mathrm{H}$ : 624.2041, found: 624.2039 .

General procedure of deacetylation. To a soln. of acetylated $O$-glycosides in $10 \mathrm{~mL} \mathrm{MeOH}$ and $2.5 \mathrm{~mL} \mathrm{H}_{2} \mathrm{O}$, was added drop- wise ammonia water (excessive), refluxed for $6 \mathrm{~h}$. After which, the mixture was evaporated and the residue was directly purified by column chromatography.

Triazole $\boldsymbol{\beta}$-D-O-glucosyl methyl benzoate (16): From 10 (24.9 $\mathrm{mg}, 0.04 \mathrm{mmol}$ ), column chromatography (EtOAc/EtOH, 1:1) afforded 16 as a white powder $(15.3 \mathrm{mg}, 87.6 \%) . R_{f}=0.50(n-$ Butanol/water/acetic acid, 3:5:1); $[\alpha]_{\mathrm{D}}=-70.0\left(c 0.1, \mathrm{CH}_{3} \mathrm{OH}\right)$; ${ }^{1} \mathrm{H}$ NMR (400 MHz, DMSO- $\left.d_{6}\right) \delta 8.22(\mathrm{~s}, 1 \mathrm{H}), 7.57-7.56(\mathrm{~m}$, 1H), 7.45 (brs, 2H), 7.24-7.23 (m, 1H), 5.04 (brs, 1H), 4.94-4.93 (m, 2H), 4.88-4.87 (m, 1H), 4.78 (brs, 2H), 4.66-4.63 (m, 1H), 4.57 (brs, $1 \mathrm{H}), 4.48$ (brs, 2H), 4.27-4.25 (m, 1H), 3.85 (s, 3H), 3.71 (brs, 1H), 3.46 (brs, 1H), 3.17-2.98 (m, 4H); ${ }^{13} \mathrm{C} \mathrm{NMR} \mathrm{(100}$ MHz, DMSO- $\left.d_{6}\right) \delta 170.0,157.8,143.8,130.9,129.9,124.7$, 121.8, 119.7, 114.6, 102.0, 76.8, 76.6, 73.3, 70.0, 66.3, 61.4, 52.0, 48.9; HRMS: calcd. for $\mathrm{C}_{19} \mathrm{H}_{25} \mathrm{~N}_{3} \mathrm{O}_{9}+\mathrm{H}: 440.1669$, found: 440.1667 .

Triazole $\beta$-D- $\boldsymbol{O}$-glucosyl methyl 5-hydroxybenzoate (17): From 11 (199.2 mg, $0.3 \mathrm{mmol}$ ), column chromatography (EtOAc/ EtOH, 1:1) afforded 17 as a white powder (141.6 mg, 97.5\%). $R_{f}=0.47$ ( $n$-Butanol/water/acetic acid, 3:5:1); $[\alpha]_{\mathrm{D}}=-105.8$ (c $\left.0.1, \mathrm{CH}_{3} \mathrm{OH}\right) ;{ }^{1} \mathrm{H}$ NMR $\left(400 \mathrm{MHz}, \mathrm{DMSO}-d_{6}\right) \delta 9.99(\mathrm{~s}, 1 \mathrm{H})$, $8.20(\mathrm{~s}, 1 \mathrm{H}), 6.99(\mathrm{~s}, 1 \mathrm{H}), 6.90(\mathrm{~s}, 1 \mathrm{H}), 6.60(\mathrm{~s}, 1 \mathrm{H}), 5.05(\mathrm{~d}$, $J=4.4 \mathrm{~Hz}, 1 \mathrm{H}), 4.95-4.94(\mathrm{~m}, 2 \mathrm{H}), 4.84(\mathrm{~d}, J=12.0 \mathrm{~Hz}, 1 \mathrm{H})$, $4.74(\mathrm{t}, J=4.0 \mathrm{~Hz}, 2 \mathrm{H}), 4.64(\mathrm{~d}, J=12.1 \mathrm{~Hz}, 1 \mathrm{H}), 4.58(\mathrm{t}, J=$ $5.7 \mathrm{~Hz}, 1 \mathrm{H}), 4.39(\mathrm{t}, J=4.3 \mathrm{~Hz}, 2 \mathrm{H}), 4.27(\mathrm{~d}, J=7.7 \mathrm{~Hz}, 1 \mathrm{H})$, $3.81(\mathrm{~s}, 3 \mathrm{H}), 3.72-3.68(\mathrm{~m}, 1 \mathrm{H}), 3.49-3.44(\mathrm{~m}, 1 \mathrm{H}), 3.17-3.13$ $(\mathrm{m}, 2 \mathrm{H}), 3.08-3.02(\mathrm{~m}, 1 \mathrm{H}), 3.00-2.95(\mathrm{~m}, 1 \mathrm{H}) ;{ }^{13} \mathrm{C}$ NMR $(100$ MHz, DMSO- $\left.d_{6}\right) \delta 171.2,164.1,163.8,149.1,136.7,129.8$, 114.3, 111.7, 110.9, 107.3, 81.8, 78.5, 75.2, 71.4, 66.5, 66.2, 56.9; HRMS: calcd. for $\mathrm{C}_{19} \mathrm{H}_{25} \mathrm{~N}_{3} \mathrm{O}_{10}+\mathrm{H}$ : 456.1618, found: 456.1618 .

Triazole $\beta$-D- $\boldsymbol{O}$-glucosyl methyl 2-hydroxybenzoate (18): From 12 (131.3 mg, $0.2 \mathrm{mmol}$ ), column chromatography (EtOAc/ EtOH, 1:1) afforded 18 as a white powder (87.9 mg, 91.9\%). $R_{f}=0.43(n-B u t a n o l /$ water/acetic acid, $3: 5: 1) ;[\alpha]_{\mathrm{D}}=-60.0(c$ $\left.0.1, \mathrm{CH}_{3} \mathrm{OH}\right) ;{ }^{1} \mathrm{H} \mathrm{NMR}\left(400 \mathrm{MHz}, \mathrm{DMSO}-d_{6}\right) \delta 10.12(\mathrm{~s}, 1 \mathrm{H})$, $8.20(\mathrm{~s}, 1 \mathrm{H}), 7.24(\mathrm{~s}, 1 \mathrm{H}), 7.15(\mathrm{~d}, J=8.4 \mathrm{~Hz}, 1 \mathrm{H}), 6.94(\mathrm{~d}, J=$ $8.8 \mathrm{~Hz}, 1 \mathrm{H}), 5.05(\mathrm{~d}, J=4.7 \mathrm{~Hz}, 1 \mathrm{H}), 5.00-4.97(\mathrm{~m}, 2 \mathrm{H}), 4.86$ $(\mathrm{d}, J=12.0 \mathrm{~Hz}, 1 \mathrm{H}), 4.72$ (brs, $2 \mathrm{H}), 4.65(\mathrm{~d}, J=12.2 \mathrm{~Hz}, 1 \mathrm{H})$, $4.60(\mathrm{t}, J=5.6 \mathrm{~Hz}, 1 \mathrm{H}), 4.36$ (brs, $2 \mathrm{H}), 4.26(\mathrm{~d}, J=7.6 \mathrm{~Hz}, 1 \mathrm{H})$, $3.88(\mathrm{~s}, 3 \mathrm{H}), 3.70(\mathrm{dd}, J=6.0 \mathrm{~Hz}, 10.4 \mathrm{~Hz}, 1 \mathrm{H}), 3.49-3.43(\mathrm{~m}$, $1 \mathrm{H}), 3.17-3.11(\mathrm{~m}, 2 \mathrm{H}), 3.08-3.03(\mathrm{~m}, 1 \mathrm{H}), 3.01-2.95(\mathrm{~m}, 1 \mathrm{H})$; ${ }^{13} \mathrm{C}$ NMR (100 MHz, DMSO- $\left.d_{6}\right) \delta 160.2,155.4,149.1,129.8$, 129.1, 123.5, 118.7, 117.4, 107.2, 81.9, 78.6, 78.5, 75.2, 72.1, $66.5,66.3,57.3,54.2$; HRMS: calcd. for $\mathrm{C}_{19} \mathrm{H}_{25} \mathrm{~N}_{3} \mathrm{O}_{10}+\mathrm{H}$ : 456.1618, found: 456.1617 .

Triazole $\beta$-D- $O$-galactosyl methyl benzoate (19): From 13 (167.8 mg, $0.3 \mathrm{mmol}$ ), column chromatography (EtOAc/EtOH, $1: 1)$ afforded 19 as a white powder (118.3 mg, 97.6\%). $R_{f}=$ 0.51 (n-Butanol/water/acetic acid, 3:5:1); $[\alpha]_{\mathrm{D}}=-63.6(c 0.1$, $\left.\mathrm{CH}_{3} \mathrm{OH}\right) ;{ }^{1} \mathrm{H}$ NMR $\left(400 \mathrm{MHz}, \mathrm{DMSO}-d_{6}\right) \delta 8.14(\mathrm{~s}, 1 \mathrm{H}), 7.59$ $(\mathrm{d}, J=7.5 \mathrm{~Hz}, 1 \mathrm{H}), 7.48$ (brs, $1 \mathrm{H}), 7.38$ (t, $J=7.9 \mathrm{~Hz}, 1 \mathrm{H})$, $7.17(\mathrm{dd}, J=2.4 \mathrm{~Hz}, 8.2 \mathrm{~Hz}, 1 \mathrm{H}), 4.93$ (d, $J=12.3 \mathrm{~Hz}, 1 \mathrm{H}), 4.79$ $(\mathrm{t}, J=4.9 \mathrm{~Hz}, 1 \mathrm{H}), 4.75(\mathrm{t}, J=12.3 \mathrm{~Hz}, 2 \mathrm{H}), 4.45(\mathrm{t}, J=4.9 \mathrm{~Hz}$, $2 \mathrm{H}), 4.27(\mathrm{~d}, J=7.5 \mathrm{~Hz}, 1 \mathrm{H}), 3.87(\mathrm{~s}, 3 \mathrm{H}), 3.77(\mathrm{~d}, J=2.9 \mathrm{~Hz}$, 1H), $3.66(\mathrm{~d}, J=5.9 \mathrm{~Hz}, 2 \mathrm{H}), 3.47-3.41(\mathrm{~m}, 3 \mathrm{H}) ;{ }^{13} \mathrm{C} \mathrm{NMR}(100$ MHz, DMSO- $\left.d_{6}\right) \delta 165.9,157.8,143.9,130.9,129.7,124.5$, $121.8,119.4,114.5,102.5,75.1,73.3,70.5,68.2,66.2,61.1$, 
60.5, 51.7, 48.9; HRMS: calcd. for $\mathrm{C}_{19} \mathrm{H}_{25} \mathrm{~N}_{3} \mathrm{O}_{9}+\mathrm{H}: 440.1669$, found: 440.1670 .

Triazole $\beta$-D- $\boldsymbol{O}$-galactosyl methyl 5-hydroxybenzoate (20): From 14 (224.8 mg, $0.4 \mathrm{mmol}$ ), column chromatography (EtOAc/ EtOH, 1:1) afforded 20 as a white powder (132.7 mg, 81.0\%). $R_{f}=0.48\left(n\right.$-Butanol/water/acetic acid, 3:5:1); $[\alpha]_{\mathrm{D}}=-101.7$ (c 0.1, $\left.\mathrm{CH}_{3} \mathrm{OH}\right) ;{ }^{1} \mathrm{H}$ NMR $\left(400 \mathrm{MHz}, \mathrm{DMSO}-d_{6}\right) \delta 9.92(\mathrm{~s}$, $1 \mathrm{H}), 8.19(\mathrm{~s}, 1 \mathrm{H}), 6.98(\mathrm{~s}, 1 \mathrm{H}), 6.91(\mathrm{~s}, 1 \mathrm{H}), 6.58(\mathrm{~s}, 1 \mathrm{H}), 4.89$ $(\mathrm{d}, J=4.5 \mathrm{~Hz}, 1 \mathrm{H}), 4.85(\mathrm{~d}, J=11.8 \mathrm{~Hz}, 1 \mathrm{H}), 4.74(\mathrm{t}, J=4.9 \mathrm{~Hz}$, $2 \mathrm{H}), 4.69$ (d, $J=4.9 \mathrm{~Hz}, 1 \mathrm{H}), 4.63-4.59$ (m, 2H), 4.40-4.38 $(\mathrm{m}, 2 \mathrm{H}), 4.36(\mathrm{~d}, J=5.1 \mathrm{~Hz}, 1 \mathrm{H}), 4.20(\mathrm{~d}, J=7.5 \mathrm{~Hz}, 1 \mathrm{H}), 3.81$ $(\mathrm{s}, 3 \mathrm{H}), 3.63-3.62(\mathrm{~m}, 2 \mathrm{H}) ;{ }^{13} \mathrm{C}$ NMR $\left(100 \mathrm{MHz}, \mathrm{DMSO}-d_{6}\right) \delta$ $165.9,158.9,158.8,143.8,131.4,124.5,109.2,106.5,105.3$, $102.5,75.1,73.2,70.5,68.1,66.1,61.2,60.4,51.7,48.8$; HRMS: calcd. for $\mathrm{C}_{19} \mathrm{H}_{25} \mathrm{~N}_{3} \mathrm{O}_{10}+\mathrm{Na}: 478.1438$, found: 478.1438 .

Triazole $\beta$-D- $O$-galactosyl methyl 2-hydroxybenzoate (21): From 15 (127.9 mg, $0.2 \mathrm{mmol}$ ), column chromatography (EtOAc/ EtOH, 1:1) afforded 21 as a white powder (132.7 mg, 89.2\%). $R_{f}=0.44\left(n\right.$-Butanol/water/acetic acid, 3:5:1); $[\alpha]_{\mathrm{D}}=-74.5(c$ $\left.0.1, \mathrm{CH}_{3} \mathrm{OH}\right) ;{ }^{1} \mathrm{H} \mathrm{NMR}\left(400 \mathrm{MHz}, \mathrm{DMSO}-d_{6}\right) \delta 10.10(\mathrm{~s}, 1 \mathrm{H})$, $8.18(\mathrm{~s}, 1 \mathrm{H}), 7.24$ (s, 1H), 7.15 (brs, 1H), 6.92 (brs, 1H), 4.88 (s, 1H), $4.86(\mathrm{~d}, J=11.3 \mathrm{~Hz}, 1 \mathrm{H}), 4.72$ (brs, 3H), 4.63 (d, $J=$ $12.1 \mathrm{~Hz}, 2 \mathrm{H}), 4.36$ (brs, 3H), 4.24-4.19 (m, $J=3.1 \mathrm{~Hz}, 1 \mathrm{H}), 3.88$ (s, 3H), 3.63 (brs, 1H), 3.53 (brs, $2 \mathrm{H}) ;{ }^{13} \mathrm{C}$ NMR (100 MHz, DMSO- $\left.d_{6}\right) \delta 168.3,158.1,143.9,124.7,124.6,118.4,113.8$, 113.5, 102.6, 75.2, 73.4, 70.4, 68.1, 66.9, 61.2, 60.5, 52.2, 48.9; HRMS: calcd. for $\mathrm{C}_{19} \mathrm{H}_{25} \mathrm{~N}_{3} \mathrm{O}_{10}+\mathrm{H}: 456.1618$, found: 456.1615 .

General procedure of saponification. To a soln. of esters in $10 \mathrm{~mL}$ THF and $2.5 \mathrm{~mL}$ water, was added $\mathrm{LiOH}$ (3 equiv.), stirring for $6 \mathrm{~h}$. The resulting mixture was acidified with $1 \mathrm{~N}$ $\mathrm{HCl}$, evaporated then purified by column chromatography.

Triazole $\boldsymbol{\beta}$-D- $\boldsymbol{O}$-glucosyl benzoate (22): From 16 (190.9 mg, $0.3 \mathrm{mmol}$ ), column chromatography (EtOAc/EtOH, 1:1 to 1:2) afforded 22 as a white powder (130.7 mg, 94.7\%). $R_{f}=0.38(n-$ Butanol/water/acetic acid, 3:5:1); $[\alpha]_{\mathrm{D}}=-95.4\left(c 0.1, \mathrm{CH}_{3} \mathrm{OH}\right)$; ${ }^{1} \mathrm{H}$ NMR $\left(400 \mathrm{MHz}, \mathrm{D}_{2} \mathrm{O}\right) \delta 7.90(\mathrm{~s}, 1 \mathrm{H}), 7.24(\mathrm{~d}, J=8.0 \mathrm{~Hz}$, 1H), 7.13-7.09 (m, 2H), 6.78 (dd, $J=2.0 \mathrm{~Hz}, 8.0 \mathrm{~Hz}, 1 \mathrm{H}), 4.59$ $(\mathrm{dd}, J=4.4 \mathrm{~Hz}, 9.2 \mathrm{~Hz}, 4 \mathrm{H}), 4.26-4.24(\mathrm{~m}, 3 \mathrm{H}), 3.62$ (dd, $J=$ $1.2 \mathrm{~Hz}, 12.4 \mathrm{~Hz}, 1 \mathrm{H}), 3.46-3.41(\mathrm{~m}, 1 \mathrm{H}), 3.22(\mathrm{t}, J=9.2 \mathrm{~Hz}$, $1 \mathrm{H}), 3.18-3.11(\mathrm{~m}, 2 \mathrm{H}), 3.04(\mathrm{dd}, J=8.0 \mathrm{~Hz}, 8.8 \mathrm{~Hz}, 1 \mathrm{H}) ;{ }^{13} \mathrm{C}$ NMR $\left(100 \mathrm{MHz}, \mathrm{D}_{2} \mathrm{O}\right) \delta 157.2,143.4,137.6,129.5,125.9$, 122.2, 117.8, 114.8, 101.2, 75.7, 75.5, 72.9, 69.4, 66.5, 62.4, 61.7, 60.5, 49.8; HRMS: calcd. for $\mathrm{C}_{18} \mathrm{H}_{23} \mathrm{~N}_{3} \mathrm{O}_{9}+\mathrm{H}: 426.1513$, found: 426.1511 .

Triazole $\boldsymbol{\beta}$-D- $\boldsymbol{O}$-glucosyl 5-hydroxybenzoate (23): From 17 (145 mg, $0.3 \mathrm{mmol}$ ), column chromatography (EtOAc/EtOH, 1:1 to 1:2) afforded $\mathbf{2 3}$ as a white powder (139.9 mg, 99.4\%). $R_{f}=0.36\left(n\right.$-Butanol/water/acetic acid, 3:5:1); $[\alpha]_{\mathrm{D}}=-75.4(c$ $0.1, \mathrm{CH}_{3} \mathrm{OH}$ ); ${ }^{1} \mathrm{H}$ NMR (400 MHz, DMSO- $\left.d_{6}\right) \delta 9.61$ (brs, $1 \mathrm{H}$ ), $8.20(\mathrm{~s}, 1 \mathrm{H}), 7.08-6.96(\mathrm{~m}, 2 \mathrm{H}), 6.44(\mathrm{~s}, 1 \mathrm{H}), 5.05(\mathrm{brs}, 1 \mathrm{H})$, 4.97 (brs, 1H), 4.86 (d, $J=12.4 \mathrm{~Hz}, 1 \mathrm{H}), 4.75$ (brs, 2H), 4.64 $(\mathrm{d}, J=12.4 \mathrm{~Hz}, 1 \mathrm{H}), 4.57$ (t, $J=5.6 \mathrm{~Hz}, 2 \mathrm{H}), 4.35$ (brs, $2 \mathrm{H})$, $4.26(\mathrm{~d}, J=8.0 \mathrm{~Hz}, 1 \mathrm{H}), 3.73-3.68(\mathrm{~m}, 1 \mathrm{H}), 3.46-3.44(\mathrm{~m}, 1 \mathrm{H})$, 3.17-3.11 (m, 2H), 3.07 (d, $J=8.4 \mathrm{~Hz}, 1 \mathrm{H}), 2.98$ (brs, $1 \mathrm{H}) ;{ }^{13} \mathrm{C}$ NMR $\left(100 \mathrm{MHz}, \mathrm{D}_{2} \mathrm{O}\right) \delta 158.6,156.7,143.7,135.4,125.9$, 109.7, 107.3, 106.2, 101.2, 75.8, 75.6, 72.9, 69.5, 66.6, 61.7, 60.6, 60.4, 49.8; HRMS: calcd. for $\mathrm{C}_{18} \mathrm{H}_{23} \mathrm{~N}_{3} \mathrm{O}_{10}+\mathrm{Na}$ : 464.1281, found: 464.1279 .
Triazole $\beta$-D- $O$-glucosyl 2-hydroxybenzoate (24): From 18 (95.6 mg, $0.2 \mathrm{mmol}$ ), column chromatography (EtOAc/EtOH, $1: 1$ to $1: 2)$ afforded 24 as a white powder $(86.2 \mathrm{mg}, 93.1 \%) . R_{f}=$ 0.29 (n-Butanol/water/acetic acid, 3:5:1); $[\alpha]_{\mathrm{D}}=-63.1(c 0.1$, $\left.\mathrm{CH}_{3} \mathrm{OH}\right) ;{ }^{1} \mathrm{H}$ NMR $\left(400 \mathrm{MHz}, \mathrm{D}_{2} \mathrm{O}\right) \delta 8.01(\mathrm{~s}, 1 \mathrm{H}), 7.18(\mathrm{~d}, J=$ $2.8 \mathrm{~Hz}, 1 \mathrm{H}), 6.87(\mathrm{dd}, J=2.8 \mathrm{~Hz}, 8.8 \mathrm{~Hz}, 1 \mathrm{H}), 6.71(\mathrm{~d}, J=9.2$ $\mathrm{Hz}, 1 \mathrm{H}), 4.82-4.70(\mathrm{~m}, 5 \mathrm{H}), 4.35-4.33(\mathrm{~m}, 2 \mathrm{H}), 3.72$ (d, $J=$ $11.6 \mathrm{~Hz}, 1 \mathrm{H}), 3.57-3.54(\mathrm{~m}, 1 \mathrm{H}), 3.30(\mathrm{t}, J=8.4 \mathrm{~Hz}, 1 \mathrm{H})$, 3.28-3.20 (m, 2H), $3.13(\mathrm{t}, J=8.0 \mathrm{~Hz}, 1 \mathrm{H})$; HRMS: calcd. for $\mathrm{C}_{18} \mathrm{H}_{23} \mathrm{~N}_{3} \mathrm{O}_{9}+\mathrm{H}$ : 442.1462 , found: 442.1485 .

Triazole $\boldsymbol{\beta}$-D- $\boldsymbol{O}$-galactosyl benzoate (25): From 19 (121.1 $\mathrm{mg}, 0.3 \mathrm{mmol}$ ), column chromatography (EtOAc/EtOH, 1:1 to $1: 2)$ afforded 25 as a white powder $(114.7 \mathrm{mg}, 97.7 \%) . R_{f}=$ 0.31 (n-Butanol/water/acetic acid, 3:5:1); $[\alpha]_{\mathrm{D}}=-72.3($ c 0.1 , $\left.\mathrm{CH}_{3} \mathrm{OH}\right) ;{ }^{1} \mathrm{H}$ NMR $\left(400 \mathrm{MHz}, \mathrm{D}_{2} \mathrm{O}\right) \delta 7.98(\mathrm{~s}, 1 \mathrm{H}), 7.38(\mathrm{~d}, J=$ $7.0 \mathrm{~Hz}, 1 \mathrm{H}), 7.19-7.16(\mathrm{~m}, 2 \mathrm{H}), 6.94(\mathrm{~d}, J=7.4 \mathrm{~Hz}, 1 \mathrm{H}), 4.78-$ 4.70 (m, 4H), 4.28 (brs, 2H), 4.19 (d, $J=7.2 \mathrm{~Hz}, 1 \mathrm{H}), 3.70$ (d, $J=2.3 \mathrm{~Hz}, 1 \mathrm{H}), 3.59-3.51(\mathrm{~m}, 2 \mathrm{H}), 3.42-3.30(\mathrm{~m}, 3 \mathrm{H}) ;{ }^{13} \mathrm{C}$ NMR $\left(100 \mathrm{MHz}, \mathrm{D}_{2} \mathrm{O}\right) \delta 157.5,143.5,131.8,129.9,126.0$, $122.8,119.9,115.2,101.6,75.1,72.6,70.5,68.5,66.5,61.5$, 60.8, 60.4, 49.9; HRMS: calcd. for $\mathrm{C}_{18} \mathrm{H}_{23} \mathrm{~N}_{3} \mathrm{O}_{9}+\mathrm{H}: 426.1513$, found: 426.1511 .

Triazole $\boldsymbol{\beta}$-D- $\boldsymbol{O}$-galactosyl 5-hydroxybenzoate (26): From 20 (112.9 mg, $0.3 \mathrm{mmol}$ ), column chromatography (EtOAc/ EtOH, $1: 1$ to $1: 2)$ afforded $\mathbf{2 6}$ as a white powder $(101.3 \mathrm{mg}$, $91.8 \%) \cdot R_{f}=0.32\left(n\right.$-Butanol/water/acetic acid, 3:5:1); $[\alpha]_{\mathrm{D}}=$ -74.2 (c 0.1, $\left.\mathrm{CH}_{3} \mathrm{OH}\right) ;{ }^{1} \mathrm{H} \mathrm{NMR}\left(400 \mathrm{MHz}, \mathrm{D}_{2} \mathrm{O}\right) \delta 8.20(\mathrm{~s}$, $1 \mathrm{H}), 7.03(\mathrm{~d}, J=7.6 \mathrm{~Hz}, 2 \mathrm{H}), 6.60(\mathrm{~s}, 1 \mathrm{H}), 5.01-4.84(\mathrm{~m}, 4 \mathrm{H})$, $4.54(\mathrm{t}, J=4.8 \mathrm{~Hz}, 2 \mathrm{H}), 4.36(\mathrm{~d}, J=7.0 \mathrm{~Hz}, 1 \mathrm{H}), 3.89(\mathrm{~d}, J=$ $1.2 \mathrm{~Hz}, 1 \mathrm{H}), 3.80-3.71(\mathrm{~m}, 2 \mathrm{H}), 3.58-3.53(\mathrm{~m}, 3 \mathrm{H}) ;{ }^{13} \mathrm{C} \mathrm{NMR}$ $\left(100 \mathrm{MHz}, \mathrm{D}_{2} \mathrm{O}\right) \delta 168.9,158.6,156.6,125.9,123.2,109.4$, 107.1, 105.3, 101.5, 75.0, 72.6, 70.5, 68.5, 66.5, 61.4, 60.8, 60.4, 49.8; HRMS: calcd. for $\mathrm{C}_{18} \mathrm{H}_{23} \mathrm{~N}_{3} \mathrm{O}_{9}+\mathrm{H}: 442.1462$, found: 442.1474

Triazole $\boldsymbol{\beta}$-D- $\boldsymbol{O}$-galactosyl 2-hydroxybenzoate (27): From 21 (93.3 mg, $0.2 \mathrm{mmol}$ ), column chromatography (EtOAc/ EtOH, $1: 1$ to $1: 2)$ afforded 27 as a white powder $(88.5 \mathrm{mg}$, $97.8 \%) . R_{f}=0.29\left(n\right.$-Butanol/water/acetic acid, 3:5:1); $[\alpha]_{\mathrm{D}}=$ -14.8 (c 0.1, $\left.\mathrm{CH}_{3} \mathrm{OH}\right) ;{ }^{1} \mathrm{H}$ NMR (400 MHz, $\left.\mathrm{D}_{2} \mathrm{O}\right) \delta 8.06(\mathrm{~s}$, $1 \mathrm{H}), 7.24(\mathrm{~d}, J=3.0 \mathrm{~Hz}, 1 \mathrm{H}), 6.93(\mathrm{dd}, J=3.0 \mathrm{~Hz}, 8.8 \mathrm{~Hz}$, $1 \mathrm{H}), 6.77(\mathrm{~d}, J=8.8 \mathrm{~Hz}, 1 \mathrm{H}), 4.88-4.75(\mathrm{~m}, 4 \mathrm{H}), 4.40$ (t, $J=$ $4.8 \mathrm{~Hz}, 2 \mathrm{H}), 4.25(\mathrm{~d}, J=7.0 \mathrm{~Hz}, 1 \mathrm{H}), 3.77(\mathrm{~d}, J=2.3 \mathrm{~Hz}, 1 \mathrm{H})$, 3.68-3.59 (m, 2H), 3.46-3.39 (m, 3H); HRMS: calcd. for $\mathrm{C}_{18} \mathrm{H}_{23} \mathrm{~N}_{3} \mathrm{O}_{9}+\mathrm{H}$ : 442.1462, found: 442.1467 .

Inhibitory assay. Recombinant human PTP1B catalytic domain was expressed and purified according to procedures described previously. ${ }^{27 a}$ Enzymatic activity of PTP1B was determined at $30{ }^{\circ} \mathrm{C}$ by monitoring the hydrolysis of $p$ NPP. Dephosphorylation of $p$ NPP generates product $p N P$, which can be monitored at $405 \mathrm{~nm}$. In a typical $100 \mu \mathrm{L}$ assay, mixture containing $50 \mathrm{mM}$ MOPS, pH 6.5, $2 \mathrm{mM} p \mathrm{NPP}$ and recombinant enzymes, PTP1B activities were continuously monitored on a SpectraMax 340 microplate reader at $405 \mathrm{~nm}$ for $2 \mathrm{~min}$ at $30^{\circ} \mathrm{C}$ and the initial rate of the hydrolysis was determined using the early linear region of the enzymatic reaction kinetic curve. For calculating $\mathrm{IC}_{50}$, inhibition assays were performed with $30 \mathrm{nM}$ recombinant enzyme, $2 \mathrm{mM} p \mathrm{NPP}$ in $50 \mathrm{mM}$ MOPS at $\mathrm{pH}$ 6.5, and the inhibitors diluted around the estimated $\mathrm{IC}_{50}$ values. $\mathrm{IC}_{50}$ 
was calculated from the nonlinear curve fitting of percent inhibition (inhibition (\%)) vs. inhibitor concentration [I] by using the following equation: inhibition $(\%)=100 /\left\{1+\left(\mathrm{IC}_{50} /[\mathrm{I}]\right) k\right\}$, where $k$ is the Hill coefficient. To study the inhibition selectivity on other PTP family members, human TCPTP, SHP1, SHP2 and LARD1 were prepared and assays were performed according to procedures described previously. ${ }^{27 \mathrm{~b}}$

\section{Result and Discussion}

Formation of the triazolyl glycosides. As illustrated in Scheme 1, the azido methyl benzoate $(\mathbf{5}, \mathbf{6}$ and $\mathbf{7})$ were prepared from commercially available methyl 3-hydroxybenzoate (2), methyl 3,5-dihydroxybenzoate (3) and methyl 2,5-dihydroxybenzoate (4) in one-pot. Etherification of 2-4 were realized in the presence of $\mathrm{K}_{2} \mathrm{CO}_{3}$ and 1,2-dibromoethane, followed by straightforward azide substitution with $\mathrm{NaN}_{3}$, furnishing the desired products 5, 6 and 7 in 47.5, 50.1 and 52.3\% yield, respectively. The known sugar alkynes $\mathbf{8}$ and $\mathbf{9}$ were synthesized according to literature procedures from readily available acetyl- $\beta$ - $O$-glucoside $\left(\mathbf{8}^{\prime}\right)$ and acetyl- $\beta$ - $O$-galactoside $\left(\mathbf{9}^{\prime}\right){ }^{26}$

With the click ingredients ready, we successively handled the microwave-assisted 1,3-dipolar cycloaddition promoted by $\mathrm{Cu}(\mathrm{I})$ (Scheme 1). This was performed in a Yalian (YL8023B1) system at $40{ }^{\circ} \mathrm{C}$ with a ramp time of $6 \mathrm{~min}$ and hold time of $8 \mathrm{~min}$. By using a condition involving 2 equiv. sodium ascorbate and 1 equiv. $\mathrm{CuSO}_{4}$ as catalyst in $1: 1 \mathrm{CH}_{2} \mathrm{Cl}_{2} / \mathrm{H}_{2} \mathrm{O}(\mathrm{v} / \mathrm{v})$ solvent mixture, the click reaction between the azides and sugar alkynes proceeded smoothly. Both glucosyl (8) and galactosyl (9) alkynes were tolerable glyco-donors in such ambiance, affording the click products 10-15 in considerable yields of $86.7-94.5 \%$. Interestingly, $\mathbf{6}$ (with meta-OH substitution on benzoate ring) represented the most efficient azido reactant which gave the

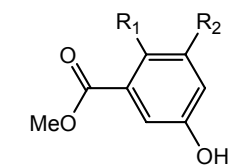

2: $\mathbf{R}_{1}=\mathrm{H}, \mathbf{R}_{\mathbf{2}}=\mathrm{H}$; 3: $R_{1}=H, R_{2}=O H$ 4: $\mathrm{R}_{1}=\mathrm{OH}, \mathrm{R}_{2}=\mathrm{H}$
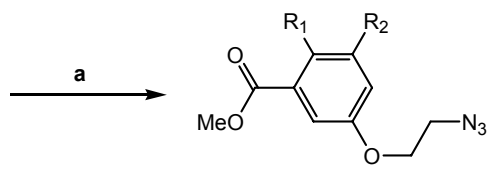

$5(48 \%): R_{1}=H, R_{2}=H$ $6(50 \%): R_{1}=H, R_{2}=\mathrm{OH}$ $7(52 \%): R_{1}=O H, R_{2}=H$

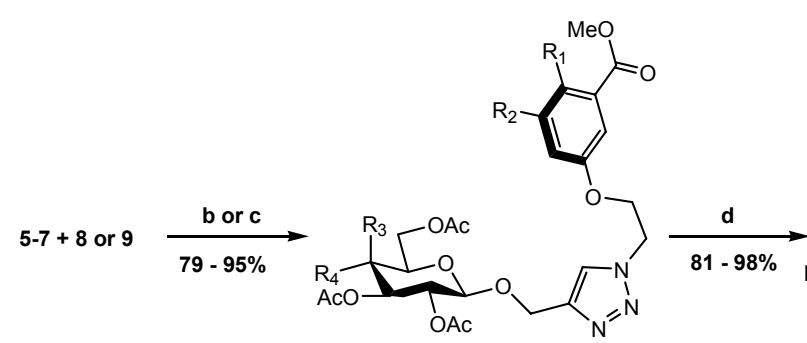

10: $R_{1}=H, R_{2}=H, R_{3}=H, R_{4}=O A C$ 11: $R_{1}=H, R_{2}=O H, R_{3}=H, R_{4}=O A C$ 12: $R_{1}=O H, R_{2}=H, R_{3}=H, R_{4}=O A c$;

13: $R_{1}=H, R_{2}=H, R_{3}=O A c, R_{4}=H$ 14: $R_{1}=H, R_{2}=O H, R_{3}=O A c, R_{4}=H$ 15: $R_{1}=O H, R_{2}=H, R_{3}=O A C, R_{4}=H$

corresponding triazole $\mathbf{1 1}$ and $\mathbf{1 4}$ in excellent yields of $\mathbf{9 3 . 6}$ and $94.5 \%$, respectively.

In order to study the predominance of the performed click reaction via microwave irradiation, we also initiated the same reactions under conventional condition (rt, stirring). The parallel results were listed in Table 1. Clearly, comparing to the microwave-accelerated synthesis, the final yields of the afforded products 10-15 did not change significantly whereas much prolonged reaction time was required $(6 \mathrm{~h})$. This experimental outcome unambiguously demonstrated the introduction of microwave irradiation into this study worthwhile, yielding the desired key products in a much faster manner.

Next, as illustrated in Scheme 1, after deacetylation with ammonia water in $\mathrm{MeOH}$, the $\mathrm{OH}$-free triazole esters 16-21 were afforded in 81.0 - 97.5\% yield. Successive saponification with $\mathrm{LiOH}$ led to the achievement of final acids 22-27 (yield: 91.8\%-quantitive).

Biological assays on PTPs. The set of freshly prepared salicylic glycosides (10-27) were assayed toward PTP1B and a panel of homologous PTPs including TCPTP, SHP-1, SHP-2

Table 1. Cu-catalyzed click reaction

\begin{tabular}{ccccccc}
\hline \multirow{2}{*}{ Azide } & Sugar & \multicolumn{2}{c}{ Microwave } & & \multicolumn{2}{c}{ Conventional } \\
\cline { 3 - 4 } \cline { 6 - 7 } & & $\begin{array}{c}\text { Time } \\
(\mathrm{min})\end{array}$ & $\begin{array}{c}\text { Product } \\
(\text { yield, \%) }\end{array}$ & & $\begin{array}{c}\text { Time } \\
(\mathrm{min})\end{array}$ & $\begin{array}{c}\text { Product } \\
(\text { yield, \%) }\end{array}$ \\
\hline $\mathbf{5}$ & & 14 & $10(87.9)$ & & 360 & $10(87.3)$ \\
$\mathbf{6}$ & $\mathbf{8}$ & 14 & $\mathbf{1 1}(\mathbf{9 3 . 6})$ & & 360 & $11(78.7)$ \\
$\mathbf{7}$ & & 14 & $12(88.0)$ & & 360 & $12(93.8)$ \\
\hline $\mathbf{5}$ & & 14 & $13(86.7)$ & & 360 & $13(74.6)$ \\
$\mathbf{6}$ & $\mathbf{9}$ & 14 & $\mathbf{1 4}(\mathbf{9 4 . 5 )}$ & & 360 & $14(92.5)$ \\
$\mathbf{7}$ & & 14 & $15(92.1)$ & & 360 & $15(94.1)$ \\
\hline
\end{tabular}
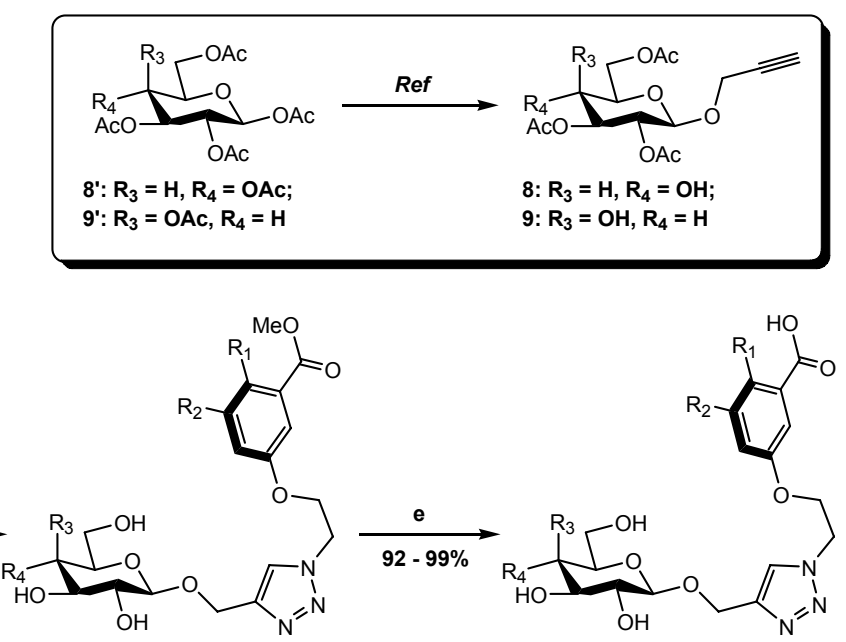

16: $R_{1}=H, R_{2}=H, R_{3}=H, R_{4}=O H$; 17: $R_{1}=H, R_{2}=O H, R_{3}=H, R_{4}=O H$; 18: $R_{1}=\mathrm{OH}, \mathrm{R}_{2}=\mathrm{H}, \mathrm{R}_{3}=\mathrm{H}, \mathrm{R}_{4}=\mathrm{OH}$ 19: $R_{1}=H, R_{2}=H, R_{3}=O H, R_{4}=H$; 20: $\mathbf{R}_{1}=\mathrm{H}, \mathrm{R}_{\mathbf{2}}=\mathrm{OH}, \mathrm{R}_{3}=\mathrm{OH}, \mathbf{R}_{\mathbf{4}}=\mathrm{H}$ 21: $R_{1}=O H, R_{2}=H, R_{3}=O H, R_{4}=H$
22: $\mathbf{R}_{\mathbf{1}}=\mathrm{H}, \mathrm{R}_{\mathbf{2}}=\mathrm{H}, \mathrm{R}_{\mathbf{3}}=\mathrm{H}, \mathbf{R}_{\mathbf{4}}=\mathrm{OH}$; 23: $R_{1}=H, R_{2}=O H, R_{3}=H, R_{4}=O H$; 24: $\mathbf{R}_{\mathbf{1}}=\mathrm{OH}, \mathrm{R}_{\mathbf{2}}=\mathrm{H}, \mathrm{R}_{\mathbf{3}}=\mathrm{H}, \mathrm{R}_{\mathbf{4}}=\mathrm{OH}$; 25: $\mathbf{R}_{1}=\mathrm{H}, \mathbf{R}_{\mathbf{2}}=\mathrm{H}, \mathbf{R}_{3}=\mathrm{OH}, \mathbf{R}_{\mathbf{4}}=\mathrm{H}$; 26: $\mathbf{R}_{1}=\mathrm{H}, \mathrm{R}_{2}=\mathrm{OH}, \mathrm{R}_{3}=\mathrm{OH}, \mathrm{R}_{4}=\mathrm{H}$; 27: $R_{1}=O H, R_{2}=H, R_{3}=O H, R_{4}=H$

Scheme 1. Reagents and Conditions: (a) $\mathrm{K}_{2} \mathrm{CO}_{3}, 1$, 2-dibromoethane in Acetone/ $\mathrm{H}_{2} \mathrm{O}\left(6: 1\right.$, v/v) then $\mathrm{NaN}_{3}$, reflux; (b) $\mathrm{VcNa} / \mathrm{CuSO}_{4}$ in $\mathrm{CH}_{2} \mathrm{Cl}_{2} /$ $\mathrm{H}_{2} \mathrm{O}$ under microwave irradiation $\left(40^{\circ} \mathrm{C}, 14 \mathrm{~min}\right.$ ); (c) $\mathrm{VcNa} / \mathrm{CuSO}_{4}$ in $\mathrm{CH}_{2} \mathrm{Cl}_{2} / \mathrm{H}_{2} \mathrm{O}$ at rt (6 h); (d) ammonia water in $\mathrm{MeOH}$, reflux; (e) $\mathrm{LiOH}$ in $\mathrm{THF} / \mathrm{H}_{2} \mathrm{O}$ 
Table 2. Inhibitory activities of synthesized glycosides

\begin{tabular}{|c|c|c|c|c|c|}
\hline \multirow{2}{*}{ Compd } & \multicolumn{5}{|c|}{ Inhibition rate- $\%\left(\mathrm{IC}_{50}-\mu \mathrm{M}\right)$} \\
\hline & PTP1B & ТСРТР & SHP-1 & SHP-2 & LAR \\
\hline 10 & $\mathrm{NA}^{b}$ & NA & NA & NA & NA \\
\hline 11 & NA & NA & NA & NA & NA \\
\hline 12 & $84.53 \pm 6.32(36.6)$ & $29.96 \pm 4.11(>160)$ & NA & NA & NA \\
\hline 13 & $54.59 \pm 3.72(>160)$ & NA & NA & NA & NA \\
\hline 14 & NA & NA & NA & NA & NA \\
\hline 15 & $73.95 \pm 10.82(66.2)$ & NA & NA & NA & NA \\
\hline 16 & $71.99 \pm 2.48(>160)$ & $24.11 \pm 4.12(>160)$ & NA & NA & NA \\
\hline 17 & NA & $26.13 \pm 6.67(>160)$ & NA & NA & NA \\
\hline 18 & $89.88 \pm 5.90(97.2)$ & $50.89 \pm 11.93(>160)$ & NA & NA & NA \\
\hline 19 & $35.27 \pm 15.23(>160)$ & NA & NA & NA & NA \\
\hline 20 & $31.15 \pm 4.01(>160)$ & NA & NA & NA & NA \\
\hline 21 & NA & NA & NA & NA & NA \\
\hline 22 & $98.26 \pm 0.79(50.5)$ & $52.74 \pm 7.48(>160)$ & NA & NA & NA \\
\hline 23 & $56.12 \pm 4.42(>160)$ & $33.94 \pm 5.01(>160)$ & NA & NA & NA \\
\hline 24 & $30.39 \pm 5.73(>160)$ & NA & NA & NA & NA \\
\hline 25 & $35.34 \pm 5.33(>160)$ & NA & NA & NA & NA \\
\hline 26 & NA & NA & $21.86 \pm 4.36(>160)$ & NA & NA \\
\hline 27 & $55.70 \pm 3.29(>160)$ & NA & NA & NA & NA \\
\hline
\end{tabular}

${ }^{a}$ Values are mean of 3 experiments at a compound concentration of $100 \mu \mathrm{g} / \mathrm{mL} ;{ }^{b}$ No activity (Inhibition rate at $100 \mu \mathrm{g} / \mathrm{mL}$ is 10 wer than $20 \%$ and $\mathrm{IC}_{50}$ is higher than $160 \mu \mathrm{M}$ ).

and LAR according to previously described procedure. ${ }^{27}$

As listed in Table 2, among the acetyl glycoside class (10-15), glucoside 12 and galactoside $\mathbf{1 5}$ displayed higher inhibitions ( 84.5 and $74.0 \%$, respectively) toward PTP1B, indicating that $\mathrm{OH}$-substitution on ortho-position of benzoate ring was spatially preferential for the inhibitory potency. In addition, the glucosyl compound 12 possessed an almost 2 -fold enhanced $\mathrm{IC}_{50}$ value $(36.6 \mu \mathrm{M})$ than that of the galactosyl compound $\mathbf{1 5}(66.2 \mu \mathrm{M})$. However, when the acetyl group was removed, the corresponding PTP1B inhibition of galactosides $\mathbf{1 9}$ and $\mathbf{2 1}$ significantly decreased. In contrary, the deacetylated glucosides 16 and 18 exhibited increased PTP1B inhibition (vs. 10 and 12, respectively) whereas the inhibitory potency of $\mathbf{1 8}\left(\mathrm{IC}_{50}=97.2 \mu \mathrm{M}\right)$ lowered moderately. Notably, the acids 23-27 which were expected as PTP1B inhibitors displayed weak inhibitions with $\mathbf{2 2}$ as an exception ( $98.4 \%$ inhibition).

As reported, the achievement of selective PTP1B inhibitors is an intractable issue. ${ }^{23,28 a}$ Obviously, all assayed compounds (Table 2) that possessed more than $50 \%$ inhibition on PTP1B $(12,13,15,16,18,22,23$ and 27) showed reasonably better inhibitions over TCPTP with no inhibitions on SHP-1, SHP-2 and LAR at $100 \mu \mathrm{g} / \mathrm{mL}$. For example, the best hit 12 owning an $\mathrm{IC}_{50}$ value of $36.6 \mu \mathrm{M}$ was at least 4 -fold more selective over other homologous PTPs tested $\left(\mathrm{IC}_{50}>160 \mu \mathrm{M}\right)$. More interestingly, compounds $12(36.6 \mu \mathrm{M})$ and $\mathbf{1 5}(66.2 \mu \mathrm{M})$ which differ only in C-4 configuration on monosaccharide moiety exhibited almost 2 -fold varied $\mathrm{IC}_{50}$ value. Such difference was also observed by comparing $\mathbf{2 2}$ which is the only acid sample having displayed measurable $\mathrm{IC}_{50}$ value $(50.5 \mu \mathrm{M}$ at $98.4 \%$ inhibition) and its C-4 epimer $\mathbf{2 5}$ (35.3\% inhibition) with the equatorial bond being privileged. This clearly demonstrated that the carbohydrate moiety contributed to the inhibitory activity and more importantly, was able to perform as desirable chiral scaffolds for probing the configurational preference of PTP1B.

Binding mode investigation. We then sought to provide a plausible explanation toward the inhibitory deficiency of the afforded acid-free salicylic glycosides (22-27, Table 2) comparing to the reported natural product $\mathbf{1}^{25}$ via docking study. We started with a crystal structure in complex with a reference ligand (PDB code: 3EB1). Water was removed from the original structure, and the rest protein was prepared using the Protein preparation wizard (Schrödinger, LLC, New York, NY, 2005). Then the compounds were docked to the active site of the protein using the Induced Fit Docking workflow (Schrödinger, LLC, New York, NY, 2005). The center atom was set to be a virtual center of referenced key residues: Phe182, Cys215 and Gly259.

Apparently, as shown in Fig. 2A, multiple hydrogen bonds were made between the salicylic precursor of the reported inhibitor 1 and the active site of PTP1B (Cys215, Arg221, Gln266, Gln262 and Ile210) which strongly indicated a typical competitive inhibition pattern. Additional hydrogen bonds were also generated between the sugar moiety and Asp48 of the YRD motif. Interestingly, the long alkyl chain attached on salicylic core concomitantly provided hydrophobic interactions with Phe182, thus preventing the closure of WPD loop, which may regard as the key factor toward the submicromole-ranged $\mathrm{IC}_{50}$ value $(0.6 \mu \mathrm{M}$ on PTP1B) of inhibitor 1 . In contrast, the inhibitor (22, Fig. 2B) synthesized in this study adopted a relatively different binding manner with PTP1B. Although the benzoic acid moiety similarly occupied the active site by making hydrogen bonds with Cys215, Arg221, Ala217, Gly218 and Ile219, however, the monosaccharide moiety tended to bind with the residue Asp265 of the second phosphotyrosine site while one additional hydrogen bond was made by the triazole ring with $\mathrm{Gln} 266$ in the same pocket. Evidently, being short of the essential long 

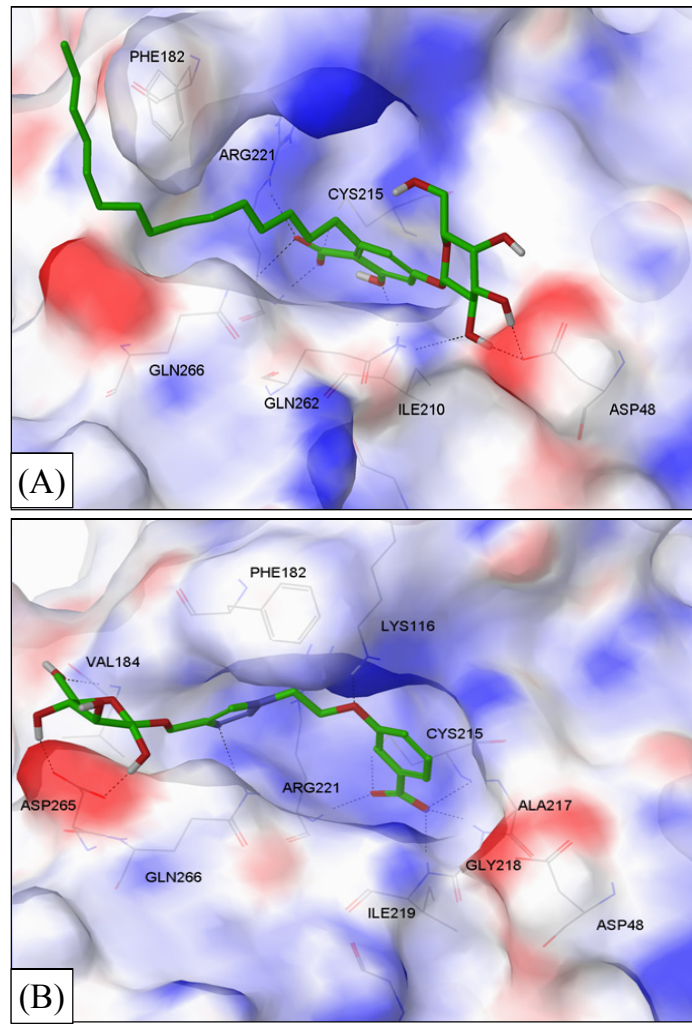

Figure 2. Binding mode of ligands ( $\mathbf{1}$ in $\mathbf{A}$ and $\mathbf{2 2}$ in $\mathbf{B}$ ) with PTP1B by docking simulation. Carbon atoms are in gray for PTP1B and green for ligands, nitrogen atoms are in blue and oxygen atoms are in red.

alkyl chain, the inhibitor $\mathbf{2 2}$ failed to make hydrophobic interactions with the phenylalanine residue Phe182, leading to the conventional WPD close conformation of PTP1B.

Consequently, we postulate the limitation of inhibitory activity of the synthesized compounds in this study is possibly resulted by the scarcity of hydrophobic functionalities conjugated with the salicylic glycosides which impedes the generation of nonpolar interactions with the WPD loop of PTP1B.

\section{Conclusions}

In conclusion, we have expeditiously prepared a series of triazole-linked salicylic $\beta$-D- $O$-glycosides via the highly efficacious microwave-accelerated click chemistry. Both glucosyl and galactosyl alkynes were tolerable glyco-donors toward such concise methodology, rapidly furnishing the desired products in high yields. The afforded products displayed promising selectivity over TCPTP, SHP-1, SHP-2 and LAR, howbeit with limited inhibitory activities on PTP1B. In addition, we have discovered that the versatile carbohydrate moiety could serve as promising chiral scaffold toward the probing of spatial preference of PTP1B. Docking study plausibly proposed binding modes of both the reported natural product 1 and synthesized compound 22 with the enzymatic target, which suggests the limited inhibitory potency of the salicylic glycosides prepared in the present study ascribable to the lack of hydrophobic functionalities on salicylate moiety to interact with Phe182 of the WPD loop. Our future efforts would thus be devoted to the preparation of sugar- based PTP1B inhibitors that simultaneously contain hydrophobic functionalities.

Acknowledgments. This work was supported by National Natural Science Foundation of China (Grant No. 20876045), National Science \& Technology Major Project of China "Key New Drug Creation and Manufacturing Program" (No. 2009 ZX09302-001), Shanghai Science and Technology Community (No. 10410702700, 08DZ2291300), and Chinese Academy of Sciences (No. KSCX2-YW-R-168). X.-P. H. also gratefully acknowledges the French Embassy in China for a doctorate fellowship and Prof. J. Xie in PPSM, ENS Cachan.

\section{Reference and Notes}

1. Bertozzi, C. R.; Kiessling, L. L. Science 2001, 291, 2357.

2. Hakomori, S. Glycoconjugate J. 2004, 21, 125.

3. Sacchettini, J. C.; Baum, L. G.; Brewer, C. F. Biochemistry 2001, 40, 3009.

4. Monsigny, M.; Mayer, R.; Roche, A. C. Carbohydr. Lett. 2000, 4, 35.

5. Feizi, T. Glycoconjugate J. 2001, 17, 553.

6. Seeberger, P. H.; Werz, D. B. Nature 2007, 446, 1046.

7. Jelinek, R.; Kolusheva, S. Chem. Rev. 2004, 104, 5987.

8. Slovin, S. F.; Keding, S. J.; Ragupathi, G. Immunol. Cell Biol. 2005, 83,418 .

9. Liang, P. H.; Wu, C. Y.; Greenberg, W. A.; Wong, C. H. Curr. Opin. Chem. Biol. 2008, 12, 86.

10. Gruner, S. A. W.; Locardi, E.; Lohof, E.; Kessler, H. Chem. Rev. 2002, 102, 491

11. Meutermans, W.; Le, G. T.; Becker, B. ChemMedChem 2006, 1, 1164.

12. Ernst, B.; Magnani, J. L. Nat. Rev. Drug Discov. 2009, 8, 661.

13. Rostovtsev, V. V.; Green, L. G.; Fokin, V. V.; Sharpless, K. B. Angew. Chem. Int. Ed. 2002, 41, 2596.

14. Mamidyala, S. K.; Finn, M. G. Chem. Soc. Rev. 2010, 39, 1252.

15. Lee, L. V.; Mitchell, M. L.; Huang, S. J.; Fokin, V. V.; Sharpless, K. B.; Wong C. H. J. Am. Chem. Soc. 2003, 125, 9588.

16. Wilkinson, B. L.; Bornaghi, L. F.; Houston, T. A.; Innocenti, A.; Vullo, D.; Supuran C. T.; Poulsen S.-A. J. Med. Chem. 2007, 50, 1651.

17. Kajimoto, T.; Node, M. Synthesis 2009, 19, 3179.

18. (a) Lin, L.; Shen, Q.; Chen, G. R.; Xie, J. Bioorg. Med. Chem. 2008, 16, 9757. (b) Deng, Q.; Zheng, R.-R.; Ding, N.-N.; He, X.-P.; Chen, G.-R. Bull. Korean Chem. Soc. 2010, 31, 1055.

19 Caddick, S.; Fitzmaurice, R. Tetrahedron 2009, 65, 3325.

20. (a) Kappe, C. O.; Van der Eycken, E. Chem. Soc. Rev. 2010, 39, 1280. (b) Xue, J.-L.; Yang, J.-W.; Deng, Q.; He, X.-P.; Chen, G.-R. Bull. Korean Chem. Soc. 2010, 31, 1825.

21. Amblard, F.; Cho, J. H.; Schinazi, R. F. Chem. Rev. 2009, 109, 4207.

22. For a very recent example, see: Dedola, S.; Hughes, D. L.; Nepogodiev, S. A.; Rejzek, M.; Field, R. A. Carbohydr. Res. 2010, 345, 1123.

23. Zhang, S.; Zhang, Z. Y. Drug Discovery Today 2007, 12, 373.

24. (a) Shrestha, S.; Bhattarai, B. R.; Lee, K.-H.; Cho, H. Bioorg. Med. Chem. 2007, 15, 6535. (b) Shrestha, S.; Lee, K.-H.; Cho, H. Bull. Korean Chem. Soc. 2004, 25, 1303.

25. Seo, C.; Sohn, J. H.; Oh, H.; Kim, B. Y.; Ahn, J. S. Bioorg. Med. Chem. Lett. 2009, 19, 6095.

26. Mereyala, H. B.; Gurrala, S. R. Carbohydr. Res. 1998, 307, 351.

27. (a) Zhang, W.; Hong, D.; Zhou, Y.-Y.; Zhang, Y.-N.; Shen, Q.; Li, J.-Y.; Hu, L.-H.; Li, J. Biochim. Biophys. Acta 2006, 1760, 1505. (b) Shi, L.; Yu, H. P.; Zhou, Y. Y.; Du, J. Q.; Shen, Q.; Li, J. Y.; Li, J. Acta Pharmacol. Sin. 2008, 29, 278.

28. Vintonyak, V. V.; Antonchick, A. P.; Rauh, D.; Waldmann, H. Curr. Opin. Chem. Biol. 2009, 13, 272-283. 